\title{
Sensitivity to Radiation-Induced Cancer in Hemochromatosis
}

(Project Number: 69939)

\section{Principal Investigators}

Richard J. Bull

Pacific Northwest National Laboratory

P.O. Box 999

Richland, WA 99352

(509) 373-6218 (phone)

Larry E. Anderson

Pacific Northwest National Laboratory

P.O. Box 999, MSIN P7-51

Richland, WA 99352

(509) 376-9635 (phone)

larry.anderson@pnl.gov 


\section{Research Objectives}

The objectives of this pilot project using HFE-knockout homozygotes and heterozygotes are to 1) determine whether the knock-out mice have greater sensitivity to radiation-induced cancer of the colon, liver and breast, 2) establish the dependence of this sensitivity on the accumulation of iron, 3) determine the extent to which cell replication and apoptosis occur in these target tissues with varying iron load, and 4) correlate the increases in sensitivity with changes in insulin-related signaling in tumors and normal tissue from each target organ.

Three experimental designs will be used in the pilot project. The sequence of experiments is designed to first explore the influence of iron load on the response and demonstrate that HFE knockout mice are more sensitive than the wild type to radiation-induced cancer in one or more of three target tissues (liver, colon and breast). The dose response relationships with a broader set of radiation doses will be explored in the second experiment. The final experiment is designed to explore the extent to which heterozygotes display the increased susceptibility to cancer induction and to independently assess the importance of iron load to the initiation versus promotion of tumors.

\section{Research Progress and Implications}

Breeding pairs of HFE knockout mice were obtained for establishing a breeding colony in our laboratory. A colony has now been established with sufficient numbers of mice to support the experimental design of this project. In addition, wild-type mice (C57BL/6J) have been obtained to serve as controls for the studies. For the first experiment, $30 \mathrm{HFE}$ knockout and 30 wild-type breeding pairs were started on a low-iron diet $(30 \mathrm{mg} / \mathrm{kg})$ on the first day of mating. Offspring from these matings have become the first experiment. Groups of 20 mice (bred and raised on the low-iron diet) will be fed purified iron diets as follows: $30,300,3000 \mathrm{mg} / \mathrm{kg}$ iron for the HFE mice and 30, 300, 3000, and 20,000 mg/kg iron for the C57BL/6J wild-type mice. These mice will be irradiated ( 1 Gy colbalt-60) at about 30 days of age. Two additional groups of nonirradiated mice (one HFE and one wild-type) will be fed the $30 \mathrm{mg} / \mathrm{kg}$ diet and used as controls.

\section{Planned Activities}

If this pilot project is successful in demonstrating substantive differences in sensitivity in one or more of these organs, a larger effort will be proposed to focus on low-dose and dose-rate effects of radiation with the intent of identifying nonlinearity and/or thresholds in the doseresponse relationships. This follow-on study would concentrate on understanding the molecular basis of interactions between Hereditary Hemochromatosis and radiation exposure. The additional work would allow us to calculate the excess risk that would be associated with low doses of radiation in the human population under EPA's draft Cancer Risk Assessment Guidelines. More important, it should provide tools that can be applied to studying this relatively large segment of the population that could have enhanced sensitivity to radiation with a focused effort in molecular epidemiology. 